\title{
Perioperative endothelin-1 levels: Searching for the hidden fingerprint of nonocclusive mesenteric ischemia
}

\author{
Giuseppe D'Ancona, MD, PhD, Stephan Kische, MD, PhD, and Hüseyin Ince, MD, PhD
}

See related article on pages 1436-42.

We commend the Saarland University Medical Centre group for its consistent effort in trying to identify the biological basis and the eventual biomarkers of nonocclusive mesenteric ischemia (NOMI) in patients undergoing cardiac surgery. ${ }^{1}$ In fact, the present publication is just the last addition to a very well-defined scientific excursus the authors have developed in the last years. ${ }^{2,3}$ It should be emphasized that despite the fact that NOMI carries a heavy burden of mortality, its existence stays poorly acknowledged, and, as an obvious consequence, NOMI often remains unrecognized and untreated.

First, in the present study, ${ }^{1}$ the authors report a $9 \%$ rate of NOMI, based on angiographic and clinical evaluation. This finding underlines the fact that in environments "tuned up" for diagnosis and treatment, NOMI occurrence is significantly high when compared with other morbidities often encountered in the perioperative phases of cardiac surgery. It is even more striking that when left untreated, NOMI has a mortality rate of more than $20 \%$. As a consequence, misdiagnosis and mistreatment of this occurrence may increase to $2 \%$ the overall mortality rate after cardiac surgery on cardiopulmonary bypass. Per se, this is an important message to the readers.

Second, we should reflect on the present tools available for NOMI diagnosis. Postoperative visceral angiography cannot be accepted, because of its invasiveness, as a standard for diagnosis. For this reason, in the near future, we should aim at "less-invasive" tools, including endothelin (ET)-1 sampling. In this context, we should review critically review the authors' findings. ${ }^{1}$ Their receiver operating characteristic (ROC) analyses of preoperative ET-1 serum levels and the occurrence of NOMI revealed an optimal cutoff value of $11.7 \mathrm{pg} / \mathrm{mL}$ with a sensitivity of $44 \%$ and a

\footnotetext{
From the Vivantes Klinikum im Friedrichshain und Am Urban, Berlin, Germany and Rostock University Medical Centre, Rostock, Germany.

Disclosures: Authors have nothing to disclose with regard to commercial support.

Received for publication Feb 19, 2015; accepted for publication Feb 20, 2015; available ahead of print March 24, 2015.

Address for reprints: Giuseppe D'Ancona, MD, PhD, Cardiovascular Clinical Research and Academic Affairs Unit, Vivantes Klinikum im Friedrichshain und Am Urban, Landsberger Allee 49, 10249 Berlin, Germany (E-mail: rgea@ hotmail.com).

J Thorac Cardiovasc Surg 2015;149:1443-4

$0022-5223 / \$ 36.00$

Copyright (C) 2015 by The American Association for Thoracic Surgery http://dx.doi.org/10.1016/j.jtcvs.2015.02.034
}

specificity of $85 \%$. ROC analyses of postoperative ET-1 serum levels showed an optimal cutoff value of $14.5 \mathrm{pg} / \mathrm{mL}$ with a sensitivity of $51 \%$ and a specificity of $94 \%$. Such a test sensitivity implies that if we were to make our diagnosis just on the basis of ET-1 sampling, we would fail to correctly diagnose NOMI in at least 1 of 2 patients (false-negative). Of note,

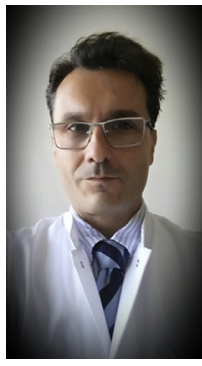
even in the authors' practice," "ET-1 is not presently used as a routine stratification tool due to the fact, that this is not a routine laboratory value and therefore cannot be provided in daily care."

Third, although we understand that Groesdonk and colleagues ${ }^{1}$ are scientifically trying to identify a specific and independent determinant for NOMI, some realistic caution should be advised. ET-1 could be just one of the many markers and mediators increasing after cardiopulmonary bypass and being involved in the ischemic-reperfusion cascade. In this context, the same group recently proposed that there is also an association between serum procalcitonin levels and NOMI. ${ }^{4}$ In the same observational cohort study of 865 patients undergoing elective cardiac surgery, ROC analyses showed that elevated serum procalcitonin levels accurately predicted the occurrence of NOMI (optimal cutoff value, $6.6 \mathrm{ng} / \mathrm{mL}$; area under the curve, 0.94; sensitivity, $71 \%$; specificity, $94 \%$ ). ${ }^{4}$ On this basis, it is reasonable to believe that the statistical correlation of ET-1 levels with the risk of developing NOMI will not necessarily imply a direct and independent causative relationship.

Fourth, the importance of other correlated risk factors and their multiplicative effect should be discussed, and the multifactorial genesis of NOMI should be clearly stated. As reported by Groesdonk and colleagues, ${ }^{1}$ the addition of 3 independent clinical risk factors (loss of sinus rhythm, need for norepinephrine $>0.1 \mu \mathrm{g} / \mathrm{kg} / \mathrm{min}$, and intra-aortic balloon pump) will determine a 7 times increase in the probability of developing NOMI. These findings should suggest to the readers that NOMI is not an "exclusive property" of cardiac surgery, and, on the contrary, it may occur and stay undiagnosed in many other patients we treat, even without the use of cardiopulmonary bypass, for other critical cardiovascular conditions.

Finally, the importance of detecting a specific role of ET1 in the genesis of NOMI has to be pictured in light of a possible future specific treatment. The fact that in animal studies ET-receptor antagonists had a protective effect in ischemia-reperfusion injury of the small bowel ${ }^{5,6}$ is 
encouraging and should stimulate further research in humans, particularly in light of the authors' present findings. ${ }^{1}$ If these initial hypotheses are confirmed, we could envisage, in the near future, a preventive treatment for patients with a higher risk of developing perioperative NOMI, on the basis of preoperatively elevated ET-1 levels.

Awareness of NOMI should be raised and its perioperative diagnosis should be simplified, achieving adequate specificity and improved sensitivity. Although the identification and diagnostic use of a single "hidden fingerprint" of intestinal ischemia are appealing, sampling of ET-1 levels should always be interpreted together with other clinical findings to further support clinicians in their daily practice. Moreover, the safety and efficacy of ET-1 antagonists in the preventive and treatment phases should be investigated in the future, within the premises of adequately sampled prospective randomized trials.

\section{References}

1. Groesdonk HV, Raffel M, Speer T, Bomberg H, Schmied W, Klingele M, et al. Elevated endothelin-1 level is a risk factor for non-occlusive mesenteric ischemia. J Thorac Cardiovasc Surg. 2015;149:1436-42.e2.

2. Groesdonk HV, Klingele M, Schlempp S, Bomberg H, Schmied W, Minko P, et al. Risk factors for nonocclusive mesenteric ischemia after elective cardiac surgery. $J$ Thorac Cardiovasc Surg. 2013;145:1603-10.

3. Bomberg H, Bierbach B, Flache S, Wagner I, Glaser L, Groesdonk HV, et al. Endothelin and vasopressin influence splanchnic blood flow distribution during and after cardiopulmonary bypass. J Thorac Cardiovasc Surg. 2013;145: 539-47.

4. Klingele M, Bomberg H, Poppleton A, Minko P, Speer T, Schäfers HJ, et al. Elevated procalcitonin in patients after cardiac surgery: a hint to nonocclusive mesenteric ischemia. Ann Thorac Surg. February 4, 2015 [Epub ahead of print].

5. Lugowska-Umer H, Umer A, Sein-Anand J, Sokolowska-Wojdylo M, Wlodarkiewicz A, Korolkiewicz RP. Endothelin receptor blockers protect against ischemia/reperfusion impairment of gastrointestinal motility in rats. Pharmacol Res. 2008;57:413-8.

6. Oktar BK, Gulpinar MA, Bozkurt A, Ghandour S, Cetinel S, Moini H, et al. Endothelin receptor blockers reduce $\mathrm{i} / \mathrm{r}$-induced intestinal mucosal injury: role of blood flow. Am J Physiol Gastrointest Liver Physiol. 2002;282:G647-55. 